EPJ Web of Conferences 87,01011 (2015)

DOI: $10.1051 /$ epjconf/20158701011

(C) Owned by the authors, published by EDP Sciences, 2015

\title{
Assessment of the ITER EC Upper Launcher Performance
}

\author{
Lorenzo Figini ${ }^{1, a}$, Daniela Farina ${ }^{1}$, Emanuele Poli², Olivier Sauter ${ }^{3}$, Alessandro Bruschi ${ }^{1}$, Timothy Goodman ${ }^{3}$, \\ Alessandro Moro ${ }^{1}$, Paola Platania ${ }^{1}$, Carlo Sozzi ${ }^{1}$, Mario Cavinato ${ }^{4}$, Gabriella Saibene ${ }^{4}$, and Mark Henderson ${ }^{5}$ \\ ${ }^{1}$ Istituto di Fisica del Plasma CNR, 20125 Milano, Italy \\ ${ }^{2}$ Max-Planck-Institut für Plasmaphysik, D-85748 Garching, Germany \\ ${ }^{3}$ EPFL-CRPP, $\mathrm{CH}-1015$ Lausanne, Switzerland \\ ${ }^{4}$ Fusion for Energy, C/ Josep Pla no 2, 08019 Barcelona, Spain \\ ${ }^{5}$ ITER Organization, Route de Vinon sur Verdon, 13115 Saint Paul Lez Durance, France
}

\begin{abstract}
The 24 MW ITER Electron Cyclotron (EC) Heating and Current Drive (H\&CD) system, operating at $170 \mathrm{GHz}$, consists of one Equatorial (EL) and four Upper Launchers (UL). The main task of the UL will be the control of Magneto-Hydrodynamic (MHD) activity such as Neoclassical Tearing Modes (NTMs) at the $\mathrm{q}=3 / 2$ and $\mathrm{q}=2$ surfaces, but it will also be needed for current profile tailoring in advanced scenarios and to assist plasma break-down and L- to H-mode transition. Moreover, it is required to be effective both when ITER will operate at nominal and reduced magnetic field magnitude.

Here the performance of the UL is assessed through the study of the full temporal evolution of different scenarios, including the reference ITER 15MA H-mode plasma, a half-field case at 2.65T, and a steady state scenario The ECCD efficiency has been evaluated for a wide range of injection angles, deriving the optimal angles and the power required for NTMs stabilization, as well as the steering range necessary to reach the rational surfaces during all the phases of the discharge. The steering sensitivity to shifts of the target or aiming errors has been estimated too. The result is an assessment of the UL design requirements to achieve the desired functionalities, which, together with the engineering limits, will be used to drive the optimization and finalization of the UL design.
\end{abstract}

\section{Introduction}

The ITER Electron Cyclotron (EC) Heating and Current Drive (H \& CD) system, operating at $170 \mathrm{GHz}$, will deliver up to $20 \mathrm{MW}$ to the plasma through one Equatorial (EL) and four Upper Launchers (UL) [1]. The capabilities foreseen for the system include central heating and bulk current drive, the control of Magneto-Hydrodynamic (MHD) activity such as Neoclassical Tearing Modes (NTMs) at the $q=3 / 2$ and $q=2$ surfaces and sawtooth instabilities at the $q=1$ surface, the assist to break-down and L- to H-mode transition phases of the plasma discharge. Moreover, it is required to be effective both when ITER will operate at nominal and reduced magnetic field magnitude. The functionalities have been partitioned between the two types of launchers, assigning the task of central heating and bulk current drive mainly to the EL, and the control of NTMs to the UL, that has consequently been designed to inject beams in the outer half of the plasma radius, focussed close to the absorption region.

The UL consists of four launchers, each housing eight beams. The eight beams follow an optical path in the launcher ending on two steering mirrors, each shared by four beams, positioned at different heights in the upper

ae-mail: figini@ifp.cnr.it port plug. The two mirrors are identified as Upper Steering Mirror (USM) and Lower Steering Mirror (LSM). The steering mechanism rotate the mirror around an axis lying on the reflecting surface, with such an orientation that the steering mainly varies the poloidal injection angle $\alpha$, keeping the toroidal injection angle $\beta$ almost constant. A switching system prior to the port entrance can deviate the 24 beams coming from the gyrotrons to any combination of the 16 entrances associated with the USM and the 16 associated with the LSM. Therefore, accounting for the transmission losses, the maximum power that can be injected from each set of steering mirrors is $P_{E C} \sim 13.3 \mathrm{MW}$.

This paper presents the analysis performed to assess the performance of the UL, and the requirements on its design to achieve all the foreseen functionalities [2]. Different scenarios have been considered, along the full temporal evolution of the discharge, including the reference ITER 15 MA H-mode plasma, a case with reduced magnetic field strength, and a steady state scenario. The plasma scenarios used for the analysis are described in Sect. 2, while the modelling framework is briefly introduced in Sect. 3 . The results regarding the performance of the UL are discussed in Sect. 4, including an assessment of the ECCD efficiency in the different scenarios, an evaluation of the power required to stabilize NTMs at the $q=3 / 2$ and $q=2$ surfaces, and a quantification of the steering range 
Table 1. Timing of the plasma discharge for the five scenarios considered in the analysis.

\begin{tabular}{lllllll}
\hline & Case 1 $(2,3)$ & & Case 6 & \multicolumn{3}{c}{ Case 8 } \\
& $t(\mathrm{~s})$ & $I_{P}(\mathrm{MA})$ & $t(\mathrm{~s})$ & $I_{P}(\mathrm{MA})$ & $t(\mathrm{~s})$ & $I_{P}(\mathrm{MA})$ \\
\hline Start & $1.3(1.3,31.3)$ & 0.5 & 1.5 & 0.5 & 9.8 & 2.2 \\
L-H transition & 80 & 15 & 32.2 & 7.5 & - & - \\
$I_{P}$ flat-top & $80-530$ & 15 & $32.2-1000$ & 7.5 & $70-2750$ & 10 \\
H-L transition & $530(743,530)$ & $15(7,15)$ & 1036 & 3.0 & - & - \\
End & $623(770,582)$ & 0.5 & 1058 & 0.25 & 2950 & 1.4 \\
\hline
\end{tabular}

necessary to reach the rational surfaces at different times in the various scenarios as well as an estimate of the accuracy required to avoid misalignment between the beam and the target or among the beams. Conclusions are given in Sect. 5.

\section{Plasma scenarios}

In order to have an overview of the expected performance of the EC system in a wide range of conditions, a total of five scenarios have been considered, along their whole time history: (i) the ITER baseline $\mathrm{H}$-mode inductive scenario, $15 \mathrm{MA}, 5.3 \mathrm{~T}, \mathrm{Q}=10$ (Case 1); (ii) a variant with the longest possible current ramp-down (Case 2); (iii) a variant with the shortest possible current ramp-up and rampdown (Case 3); (iv) a half-field scenario, 7.5 MA, 2.65 T (Case 6); and (v) a steady-state scenario, $10 \mathrm{MA}, 5.3 \mathrm{~T}$ (Case 8). The purpose of studying Cases 2 and 3 is to assess the impact of different strategies for starting and ending the plasma discharge on the UL capabilities. The analysis of Case 6 is fundamental to have a good knowledge of the EC system behaviour in the first phases of the ITER operations, when it will run with reduced magnetic field strength. Finally, for Case 8 the focus will be more on pure ECCD performance than NTM stabilization efficiency, since in this advances scenario the low order $q=2$ and $q=3 / 2$ rational surfaces will be closer to the plasma center, and the main tasks of the EC system will be bulk CD and current profile tailoring. The different phases of these scenarios, including the duration of the current ramps and flat-top, and the time at which the transitions from $\mathrm{L}$ - to $\mathrm{H}$ - and $\mathrm{H}$ - to L-mode confinement occurs, are indicated in table 1.

The main parameters relevant for the evaluation of ECCD and NTM stabilization efficiency on the $q=2$ and $q=3 / 2$ surfaces are reported in tables 2 and 3 respectively, for the Cases 1, 6 and 8 at the end of the current flattop (Cases 2, 3 are identical to Case 1 in that phase). For the same time values figure 1 shows the profiles of electron temperature $T_{e}$, density $n_{e}$ and safety factor $q$ in the three scenarios. We can already anticipate here, looking at tables 2 and 3, and at figure 1, that NTM stabilization efficiency will be higher in the half-field scenario with respect to the baseline case, thanks to the more favourable $T_{e} / n_{e}$ ratio and to the lower value of bootstrap current $J_{B S}$. The highest ECCD efficiency can be expected in the steadystate scenario, which shows the largest $T_{e} / n_{e}$ ratio, but at the same time more current needs to be driven for NTM stabilization given the large $J_{B S}$ in this case.
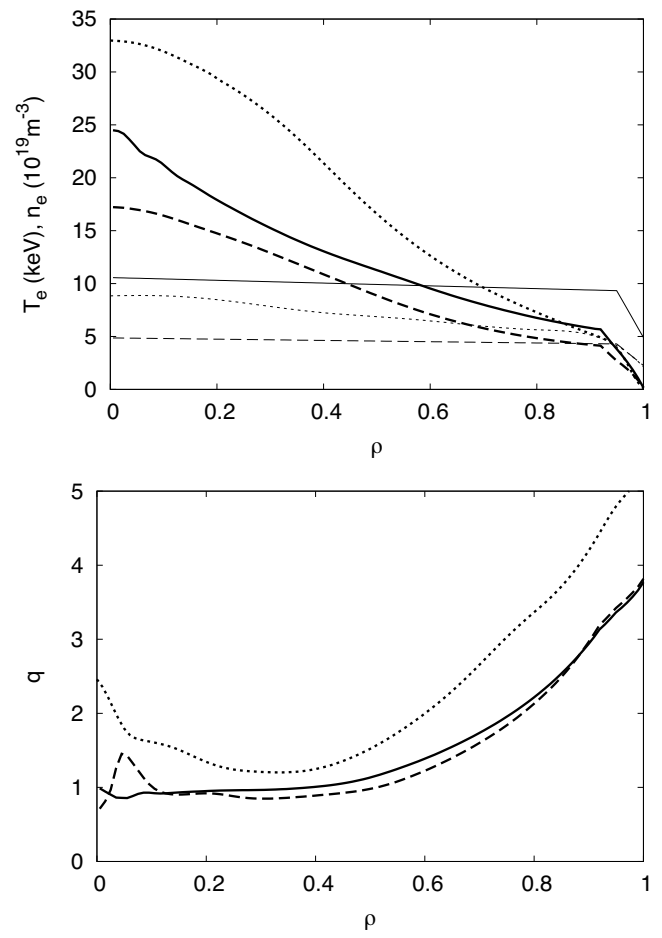

Figure 1. Top: radial profiles of electron temperature $T_{e}$ (thick) and density $n_{e}$ (thin) at the end of the current flat-top of baseline (solid), half-field (dashed), and steady-state (dotted) scenarios. Bottom: safety factor $q$.

Table 2. Plasma parameters on the $q=2$ surface, end of the current flat-top.

\begin{tabular}{lccc}
\hline & Case 1 & Case 6 & Case 8 \\
& $t=520 \mathrm{~s}$ & $t=820 \mathrm{~s}$ & $t=2450 \mathrm{~s}$ \\
\hline$\rho$ & 0.759 & 0.779 & 0.600 \\
$n_{e}\left(10^{19} / \mathrm{m}^{3}\right)$ & 9.58 & 4.40 & 6.47 \\
$T_{e}(\mathrm{keV})$ & 7.23 & 5.01 & 12.62 \\
$J_{B S}\left(\mathrm{kA} / \mathrm{m}^{2}\right)$ & 74.4 & 33.0 & 215.1 \\
\hline
\end{tabular}

Table 3. Plasma parameters on the $q=3 / 2$ surface, end of the current flat-top.

\begin{tabular}{lccc}
\hline & Case 1 & Case 6 & Case 8 \\
& $t=520 \mathrm{~s}$ & $t=820 \mathrm{~s}$ & $t=2450 \mathrm{~s}$ \\
\hline$\rho$ & 0.636 & 0.676 & 0.494 \\
$n_{e}\left(10^{19} / \mathrm{m}^{3}\right)$ & 9.74 & 4.46 & 6.89 \\
$T_{e}(\mathrm{keV})$ & 8.95 & 6.06 & 16.85 \\
$J_{B S}\left(\mathrm{kA} / \mathrm{m}^{2}\right)$ & 81.8 & 39.2 & 216.5 \\
\hline
\end{tabular}


Table 4. Launching coordinates and beam parameters.

\begin{tabular}{lcccc}
\hline Mirror & $R(\mathrm{~m})$ & $z(\mathrm{~m})$ & $w_{0}(\mathrm{~m})$ & $d_{0}(\mathrm{~m})$ \\
\hline USM & 6.999 & 4.414 & 0.029 & 2.134 \\
LSM & 7.054 & 4.178 & 0.021 & 1.620 \\
\hline
\end{tabular}

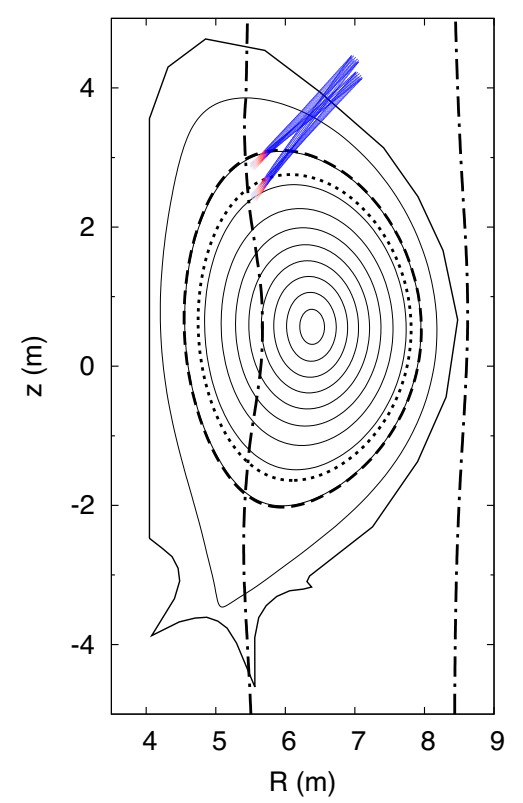

Figure 2. Beam tracing from USM and LSM aiming at the $q=2$ (dashed) and $q=3 / 2$ (dotted) surfaces for the half-field scenario at $t=820 \mathrm{~s}$. The first and second harmonic cold resonances (dash-dot) are visible outside the plasma and on the high field side of the magnetic axis respectively.

\section{Modelling framework}

All the calculations of ECH\&CD made for the analysis presented here have been performed with the EC beamtracing codes GRAY [3], which includes momentum conservation in the calculation of the driven current [4].

For the sake of simplicity, a single stigmatic "virtual" beam has been used for each of the USM and of the LSM. The launching coordinates $R$ and $z$, the beam waist $w_{0}$ and the focal distance $d_{0}$, i.e. the distance between the injection point and the beam waist for propagation in vacuum, are shown in table 4 for the two mirrors. The actual incidence points of the beams on the steering mirrors are spread along the steering axis, symmetrically around the launching point considered. Such a small spatial shift $(\delta R<0.02 \mathrm{~m}, \delta z<0.03 \mathrm{~m})$ does not alter ECCD performance significantly, the main effect being the need to adjust the injection angles by fractions of degree to recover the same results. For the same reason the launching coordinates have been considered constant for varying poloidal $\alpha$ and toroidal $\beta$ injection angles.

For all the scenarios except the half-field case, injection of ordinary mode polarized waves has been considered. For the reduced-field case extraordinary mode injection has been assumed instead, since interaction occurs at the second harmonic (see figure 2).
The expressions used for the estimate of the NTM stabilization power that will be used in Sect. 4.2 involve the ratio between the peak value of the EC driven current density $J_{C D}$ and the bootstrap current density $J_{B S}$ at the rational surface. For full consistency, the same definition has been used for $J_{C D}$ as that used for $J_{B S}$ in the different scenarios: $J_{C D}=\left\langle\mathbf{J}_{C D} \cdot \mathbf{B}\right\rangle / B_{\text {ref }}$, with $B_{r e f}=\langle B\rangle$ for all the cases except Case 8 , in which $B_{\text {ref }}=5.3 \mathrm{~T}$. The reconstruction of the EC current and power density profiles as computed in ray/beam tracing codes is somewhat arbitrary in specific conditions like, e.g., when the beam trajectory is almost tangent to a magnetic surface. A suitable area/volume preserving procedure is already applied to smooth the profiles in the GRAY code, that provides a proper profile characterization on a chosen radial grid both in the case of Gaussian-like and irregular profiles. However, for a more robust estimate, the definition

$$
J_{P}=\frac{2}{\sqrt{\pi} \Delta \rho} \frac{I_{C D}}{(d A / d \rho)_{\langle\rho\rangle_{J}}}
$$

has been introduced for the peak current density in this work, that corresponds to the peak value of a Gaussian profile centered at

$$
\langle\rho\rangle_{J}=\frac{\int d A \rho\left|J_{C D}(\rho)\right|}{\int d A J_{C D}(\rho)},
$$

with full width $\Delta \rho$ and total driven current $I_{C D}$. The definition of $J_{P}$ provides the same peak value as the standard procedure whenever the profiles are "regular", while it provides an appropriate Gaussian average value in case of "spiky" profiles. Here and in the rest of the paper we use as flux label the radius $\rho$ being the squared root of the toroidal flux normalized at the plasma edge. The minor radius of the plasma has been considered to be $a=2 \mathrm{~m}$.

\section{Upper Launcher performance}

The ECCD performance has been characterized in detail along the full time history of the five scenarios. A wide range of poloidal and toroidal launching angles has been considered to analyze the performance end of the current flat-top phase, namely at $t=520 \mathrm{~s}, t=820 \mathrm{~s}$, and $t=2450 \mathrm{~s}$ for the baseline, half-field, and steadystate scenario respectively, to have a clear overview of the ECCD efficiency around the nominal toroidal injection angle $\beta=20^{\circ}$ and across a large fraction of the plasma cross section. NTM stabilization efficiency at the $q=2$ and $q=3 / 2$ rational surfaces has been evaluated along the time evolution of the plasma discharges. NTM stabilization is a primary objective for the baseline and the halffield scenarios, while the internal position of the two rational surfaces in the steady-state case makes NTMs are a minor concern in this case. However high CD efficiency is a strong requirement also for this scenario, for current profile tailoring,

\subsection{ECCD efficiency at end of flat-top}

To evaluate ECCD efficiency at the end of the current flattop, the injection angles have been varied in the range 

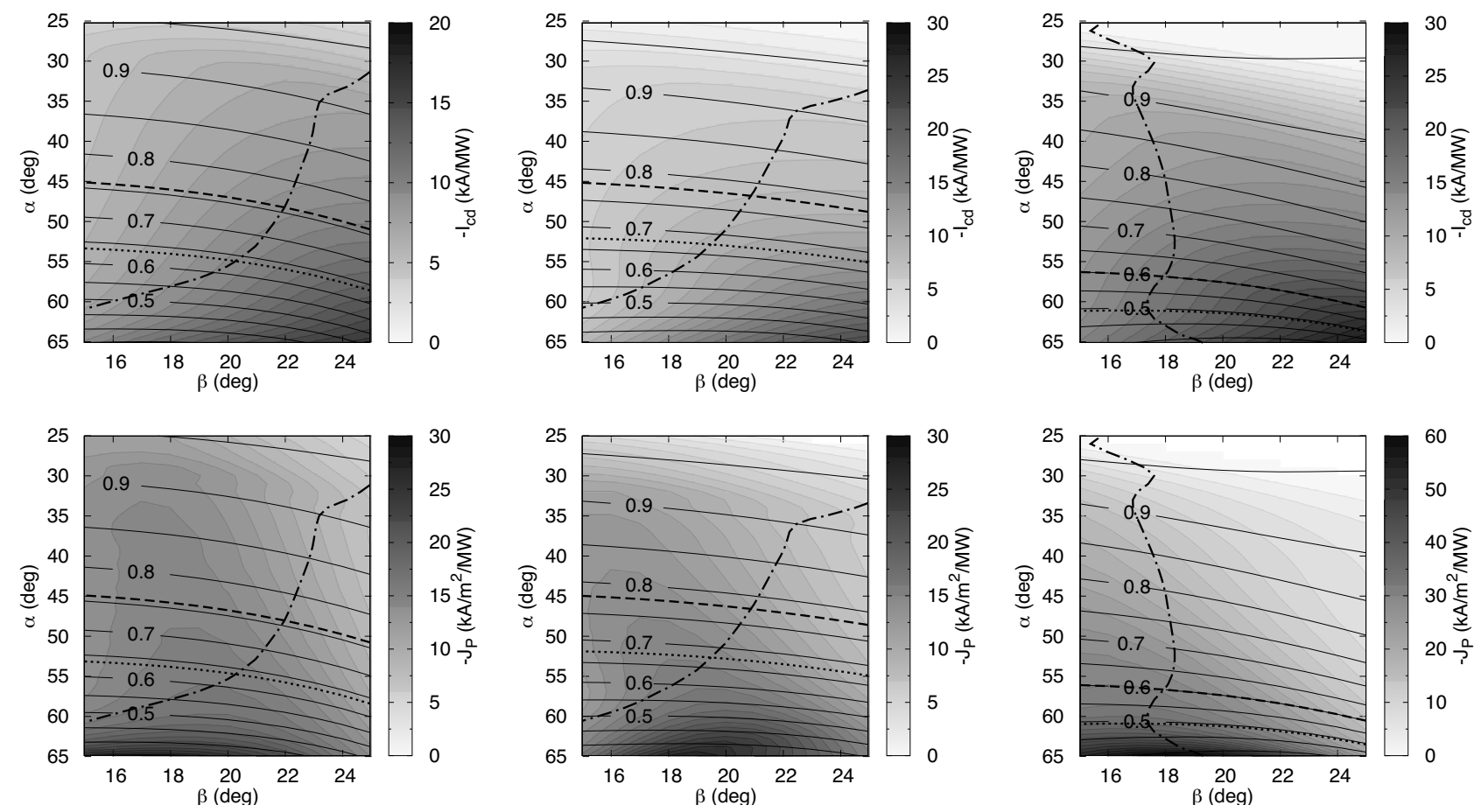

Figure 3. EC driven current (top) and peak current density (bottom) per unit injected power (shaded contours) and radial location (labelled black contours) versus toroidal $\beta$ and poloidal $\alpha$ injection angles from USM for baseline (left), half-field (center), and steadystate (right) scenarios at the end of the current flat-top. The injection angles required to drive current at the $q=2$ (dashed) and $q=3 / 2$ (dotted) surfaces, and those giving a current profile width $\Delta \rho=0.025$ (dash-dot) are shown.
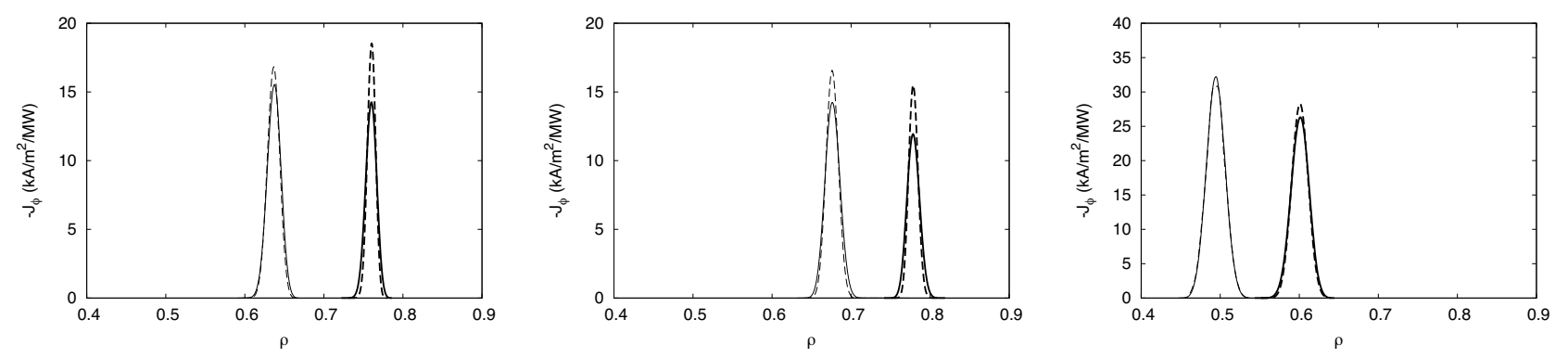

Figure 4. Toroidal driven current density profiles $J_{\phi}$ at the $q=2$ (thick) and $q=3 / 2$ (thin) surfaces with $\beta=20^{\circ}$, from USM (solid) or LSM (dotted), for baseline (left), half-field (centre), and steady-state (right) scenarios at the end of the current flat-top.

$25^{\circ} \leq \alpha \leq 65^{\circ}$, and $15^{\circ} \leq \beta \leq 25^{\circ}$, and the results for the USM are shown in figure 3 , where the driven current $I_{C D}$, the peak current density $J_{P}$, and the driven current radial location $\langle\rho\rangle_{J}$ are represented as a function of the injection angles. The toroidal current density $J_{\phi}$ driven when aiming at the $q=2$ and $q=3 / 2$ rational surfaces is shown in figure 4. As expected, at a given radius $\rho$ the driven current $I_{C D}$ and the current profile width $\Delta \rho$ increase with increasing toroidal injection angle, while the peak current density $J_{P}$ has a weak dependence on $\beta$ with a broad maximum for both mirrors in the range $17^{\circ}<\beta<20^{\circ}$ for in the radial region of interest for NTMs. The LSM delivers larger $J_{P}$ and smaller $\Delta \rho$ than the USM for a given $\beta$, mainly due to the smaller size of the beam, while the total current $I_{C D}$ is approximately the same.
Cases 1 and 6 show a similar general behaviour, with rational surfaces approximately at the same radial location, maximum $J_{P}$ at similar values of $\beta$ and slightly larger $\Delta \rho$. $\mathrm{CD}$ efficiency is marginally higher in the half-field case, due to the more advantageous $T_{e} / n_{e}$ ratio. The different shape of the $T_{e}$ and $n_{e}$ profiles for the steady-state scenario, with even higher $T_{e} / n_{e}$ ratio in the outer plasma region, allows to obtain a driven current $I_{C D}$ about 1.5 times higher than Cases 1 and 6 at given $\rho$, but also larger $\Delta \rho$ at $\rho>0.6$.

\subsection{Power requirements for NTM stabilization}

The power required for NTM stabilization has been estimated making use of two stabilization criteria [6-8]. The first criterion is expressed in terms of the ratio between the peak value of the current density $J_{C D}$ driven by the in- 


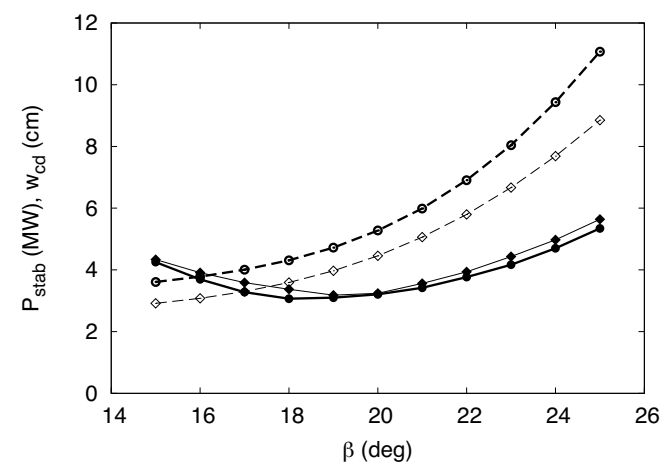

Figure 5. Power $P_{\text {stab }}$ (solid) required for NTM stabilization at $q=2$ (thick) and $q=3 / 2$ (thin) surfaces, and driven current profile width $w_{C D}$ (dashed), as a function of the toroidal launching angle $\beta$. Case $6, t=820 \mathrm{~s}$, injection from the USM.

jected EC wave and the bootstrap current density $J_{B S}$, and reads

$$
\eta_{N T M}=\frac{J_{C D}}{J_{B S}}>1.2,
$$

while the second criterion is related to the total driven current $\left(\sim J_{C D} w_{C D}\right)$ and reads

$$
\eta_{N T M} w_{C D}>0.05 \mathrm{~m}, \quad \text { for } w_{C D}<0.05 \mathrm{~m}
$$

The first criterion is valid in the limit of current profile $w_{C D}$ large with respect to the marginal size of the NTM island, the second in the opposite limit, and the two coincide when $w_{C D} \sim 0.042 \mathrm{~m}$. Defining $\eta_{N T M, 1}$ the driven current density ratio of (3) obtained for an injected power $P=1 \mathrm{MW}$, two values for the power required for NTM stabilization can be obtained from the above expressions:

$$
P>P_{\eta}=1.2 \frac{1 \mathrm{MW}}{\eta_{N T M, 1}}
$$

and

$$
P>P_{\eta w}=\frac{0.05 \mathrm{~m}}{w_{C D}} \frac{1 \mathrm{MW}}{\eta_{N T M, 1}}
$$

respectively. For the analysis performed here, the power required for NTM stabilization is estimated as the maximum between the values provided by the two criteria $P_{\text {stab }}=\max \left(P_{\eta}, P_{\eta, w}\right)$.

Due to the dependence of $w_{C D}$ and $J_{C D}$ on $\beta$ described in Sect. 4.1, $P_{\eta w}$ decreases with increasing $\beta$, while $P_{\eta}$ increases. $P_{\text {stab }}=P_{\eta}$ for sufficiently large $\beta, P_{\text {stab }}=P_{\eta w}$ at small toroidal injection angles, and the power required at a given rational surface is minimized for a value of $\beta$ for which $w_{C D} \sim 0.042 \mathrm{~m}$. This dependence on the toroidal injection angle is shown in figure 5 for the USM in the halffield scenario. The minimum of $P_{\text {stab }}$ at the $q=2$ surface is obtained for an angle $\beta \sim 19^{\circ}$, with $P_{\text {stab }}=3.2 \mathrm{MW}$, the optimal angle being slightly smaller at the $q=3 / 2$ surface, with similar value of $P_{\text {stab }}$.

The baseline scenario requires higher power levels, about 6.5 MW at the $q=2$ surface for the USM, due to both smaller $T_{e} / n_{e}$ ratio, and higher bootstrap current. Tables 5 and 6 summarize the values obtained in the three
Table 5. Power $P_{s, \min }$ required at the end of flat-top for NTM stabilization from the USM at the optimal angle $\beta_{\min }$, and power $P_{s, 20}$ required and current profile width $w_{C D, 20}$ at $\beta=20^{\circ}$.

\begin{tabular}{lllll}
\hline & $\begin{array}{l}P_{s, \min } \\
(\mathrm{MW})\end{array}$ & $\begin{array}{l}\beta_{\min } \\
\left({ }^{\circ}\right)\end{array}$ & $\begin{array}{l}P_{s, 20} \\
(\mathrm{MW})\end{array}$ & $\begin{array}{l}w_{C D, 20} \\
(\mathrm{~cm})\end{array}$ \\
\hline Case $1, q=2$ & 6.5 & 21 & 6.6 & 3.9 \\
Case $1, q=3 / 2$ & 6.1 & 19 & 6.2 & 4.9 \\
Case $6, q=2$ & 3.2 & 19 & 3.2 & 4.5 \\
Case $6, q=3 / 2$ & 3.1 & 18 & 3.2 & 5.3 \\
Case $8, q=2$ & 8.3 & 16 & 9.4 & 6.3 \\
Case $8, q=3 / 2$ & 7.2 & 16 & 8.0 & 6.6 \\
\hline
\end{tabular}

Table 6. Same as table 5, for LSM.

\begin{tabular}{lllll}
\hline & $\begin{array}{l}P_{s, \min } \\
(\mathrm{MW})\end{array}$ & $\begin{array}{l}\beta_{\min } \\
\left.{ }^{\circ}\right)\end{array}$ & $\begin{array}{l}P_{s, 20} \\
(\mathrm{MW})\end{array}$ & $\begin{array}{l}w_{C D, 20} \\
(\mathrm{~cm})\end{array}$ \\
\hline Case $1, q=2$ & 6.0 & 23 & 6.7 & 2.9 \\
Case $1, q=3 / 2$ & 5.7 & 20 & 5.7 & 4.4 \\
Case $6, q=2$ & 3.0 & 22 & 3.2 & 3.3 \\
Case $6, q=3 / 2$ & 2.8 & 20 & 2.8 & 4.4 \\
Case $8, q=2$ & 7.8 & 17 & 8.8 & 5.8 \\
Case $8, q=3 / 2$ & 7.6 & 17 & 8.1 & 6.7 \\
\hline
\end{tabular}

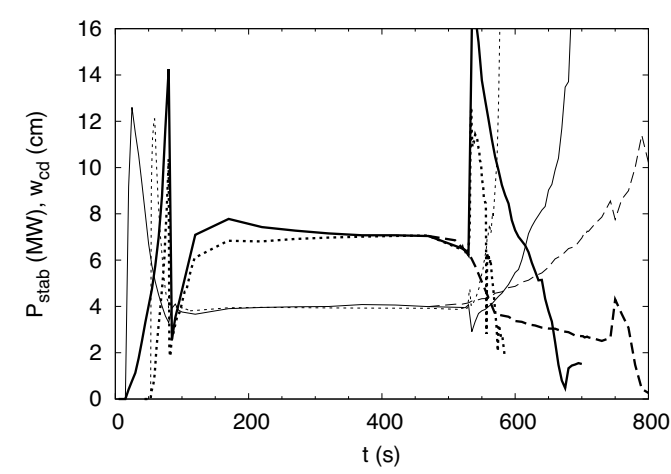

Figure 6. Power $P_{\text {stab }}$ (thick) required for NTM stabilization at the $q=2$ surfaces, and driven current profile width $w_{C D}$ (thin), during the plasma discharge of Cases 1 (solid), 2 (dashed), and 3 (dotted). Injection from the USM with $\beta=20^{\circ}$.

scenarios for both the steering mirrors. The high power required in the steady-state scenario despite the high $C D$ efficiency is easily explained with the large bootstrap current present in this scenario. It can also be noted that the minimum $P_{\text {stab }}$ is reached at a smaller angle $\beta$ than the other scenarios due to the larger profile width $w_{C D}$ obtained at a given $\beta$. The optimal toroidal injection angle minimizing $P_{\text {stab }}$ is in the range $16^{\circ} \leq \beta_{\text {min }} \leq 23^{\circ}$, depending on scenario, steering mirror and rational surface considered, with only marginally larger requirements at the nominal value $\beta=20^{\circ}$.

The evolution of the power $P_{\text {stab }}$ required along the time history of the variants of the baseline scenario is visible in figure 6. Stabilization power is almost constant during the current flat-top, but it shows large peaks at the L-H and $\mathrm{H}-\mathrm{L}$ transitions where it exceeds the maximum nominal available power $\mathrm{PEC}=13.3 \mathrm{MW}$, the increase of $P_{\text {stab }}$ being related to a sharp variation of the electron temper- 


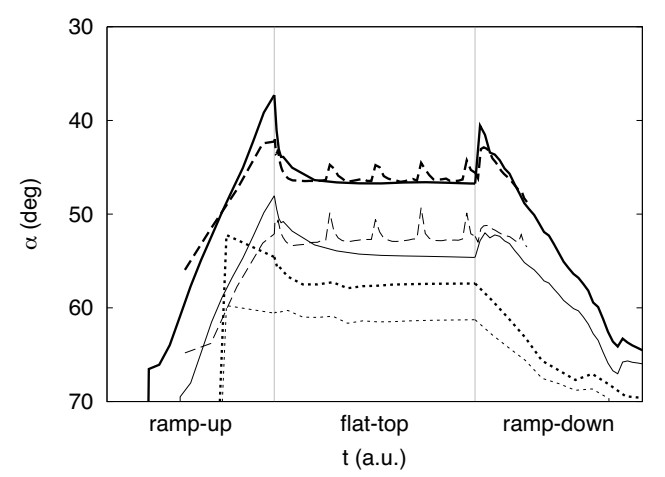

Figure 7. Poloidal injection angle $\alpha$ necessary to aim at the $\mathrm{q}=2$ (thick) and $\mathrm{q}=3 / 2$ (thin) surfaces, along the time history of the baseline (solid), half-field (dashed), and steady-state (dotted) scenarios. Injection from the USM with $\beta=20^{\circ}$. Time axis is not in scale.

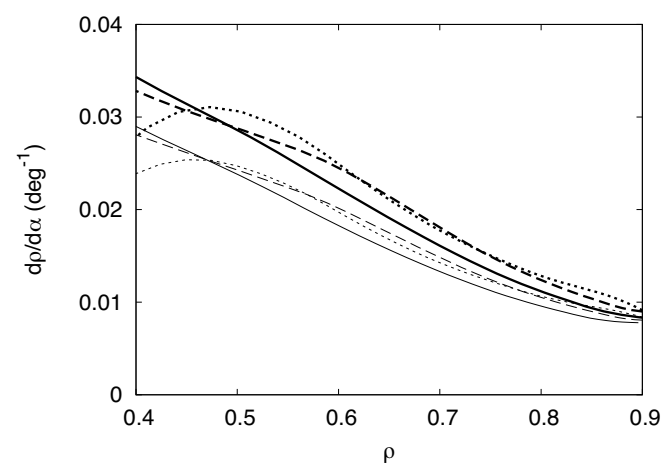

Figure 8. Steering sensitivity $|d \rho / d \alpha|$ of USM (thick) and LSM (thin) at the end of the current flat top of the baseline (solid), halffield (dashed), and steady-state (dotted) scenarios for $\beta=20^{\circ}$.

ature. The same occurs in the half-field scenario, while the late H-L transition of Case 2, and the lower $T_{e}$ drop at the $\mathrm{q}=3 / 2$ surface allow to remain within the limits of available power. However it should be noted that all these scenarios have been modelled with a prescribed time evolution of the plasma density profile, possibly giving a nonrealistic picture.

\subsection{Steering requirements}

A brief analysis of the requirements on steering range and accuracy has been performed too. In figure 7, the poloidal angle $\alpha$ necessary to aim the USM at the two rational surfaces is plotted versus the time history of the plasma discharge, for the reference toroidal angle value $\beta=20^{\circ}$. The range $\Delta \alpha=24^{\circ}$ allowed by the steering mechanism is sufficient to aim at both $q=2$ and $q=3 / 2$ surfaces in all the relevant phases of all the scenarios, however there is small margin: the range must be centered accurately to allow use of the UL also during transient events and for the other desirable tasks, such as current profile tailoring or sawtooth control.

The steering sensitivity has been assessed by computing the derivative of the driven current radial location $\rho$ with respect to the injection angle $\alpha$. The values obtained at the end of the current flat-top are shown in figure 8, and are in the range $0.01 \mathrm{deg}^{-1} \lesssim|d \rho / d \alpha| \lesssim 0.03 \mathrm{deg}^{-1}$. From this quantity a few estimates can be made. One of these is the radial shift $\delta_{1}=a|d \rho / d \alpha| 1^{\circ}$ experienced by the driven current profile under a change of the injection angle $\alpha$ by $1^{\circ}$. Its value varies in the range $0.02 \mathrm{~m} \lesssim \delta_{1} \lesssim 0.06 \mathrm{~m}$ depending on the radial position and on the steering mirror considered, and it increases with decreasing $\rho$, so that it is larger on the $q=3 / 2$ surface. The different injection geometry makes the USM more sensitive to changes of the poloidal injection angle, so that a tighter tolerance on $\alpha$ is necessary but a smaller steering range is sufficient to cover the same radial extent.

Considering that two Gaussian profiles of width $w_{C D}$ are misaligned if they are separated by an amount $\delta>\delta_{m}=$ $w_{C D} / \sqrt{2}$, a second quantity can be introduced to describe the tolerance on the convergence of multiple beams on the same target: two beams that under ideal conditions deliver perfectly overlapped current profiles become misaligned when the injection angle deviates from the intended value by more than $\Delta \alpha_{m}=w_{C D} /(\sqrt{2} a|d \rho / d \alpha|)$. For the baseline scenario at the end of the current flat-top a typical value of $\Delta \alpha_{m} \sim 1^{\circ}$ is found for both USM and LSM.

\section{Conclusions}

The analysis of a varied set of ITER scenarios along all their time history allowed to have a clear overview of the UL performance that can be expected in the different phases of ITER operation, though a finer modelling of the transient phases in the scenarios would be help confirming the capabilities of the EC system. The steering range and the available power appear adequate to fulfill the foreseen tasks, and the steering sensitivity assessment can be useful in the finalization of the UL design.

\section{Acknowledgements}

This work was supported by Fusion for Energy under the grant contract F4E-2010-GRT-161. The views and opinions expressed herein reflect only the author's views. Fusion for Energy is not liable for any use that may be made of the information contained therein.

\section{References}

[1] M.A. Henderson et al., Nuclear Fusion 48, 054013 (2008)

[2] D. Farina et al., Physics of Plasmas , in press (2014)

[3] D. Farina, Fusion Science and Technology 52, 154 (2007)

[4] N.B. Marushchenko, J. Plasma Fusion Res 2, S1000 (2007)

[5] D. Farina et al., Nuclear Fusion 52, 033005 (2012)

[6] H. Zohm et al., Plasma Physics and Controlled Fusion 49, B341 (2007)

[7] O. Sauter et al., Plasma Physics and Controlled Fusion 52, 025002 (2010)

[8] N. Bertelli et al., Nuclear Fusion 51, 103007 (2011) 